\title{
Strange / anti-strange asymmetry in the nucleon sea
}

\author{
H. R. Christiansen* and J. Magnin ${ }^{\dagger}$ \\ Centro Brasileiro de Pesquisas Físicas, CBPF - DCP \\ Rua Dr. Xavier Sigaud 150, 22290-180, Rio de Janeiro, Brazil \\ To appear in Phys. Lett. B
}

\begin{abstract}
We analyze the non-perturbative structure of the strange sea of the nucleon within a meson cloud picture. In a low $Q^{2}$ approach in which the nucleon is viewed as a three valon bound state, we evaluate the probability distribution of an in-nucleon Kaon-Hyperon pair in terms of splitting functions and recombination. The resulting kaon and hyperon probability densities are convoluted with suitable strange distributions inside the meson and baryon in order to obtain non-perturbative contributions to the strange sea of the nucleon. We find a structured strange/anti-strange asymmetry, displaying a clear excess of quarks (anti-quarks) for large (small) momentum fractions.
\end{abstract}

\section{Motivation}

According to the Quark Parton Model, with its subsequent improvements coming from Quantum Chromodynamics, it is known that hadrons are built up from a fixed number of valence quarks plus a fluctuating number of gluons and sea quark anti-quark pairs. As the momentum scale rises up, the nucleon's sea is mainly perturbatively generated and consequently quarks and anti-quarks have the same probability and momentum distributions. However, a small fraction of the sea may be associated with non-perturbative processes raising the possibility of generating unequal quark and anti-quark distributions. For instance, a non-perturbative $q-\bar{q}$ asymmetry in the charmed sea of the nucleon could play an important role in explaining the excess of events at large $x$ and $Q^{2}$ in $e^{+} p$ neutral and charged current deep inelastic scattering [1], as seen at HERA by H1 [2] and ZEUS [3]. Regarding the strange nucleon's sea, the present status of the experimental data does not exclude

*e-mail: hugo@cat.cbpf.br

†e-mail: jmagnin@lafex.cbpf.br 
the possibility of asymmetric $s$ and $\bar{s}$ distributions. Actually, it is an important question related to current experimental research. Recently, the CCFR Collaboration has analyzed the strange quark distribution in nucleons allowing explicitly for $s(x) \neq \bar{s}(x)$ distributions [4] (see also the CTEQ analyses [5]). Although the analysis of CCFR seems to indicate a rather small asymmetry, the error bars are still too large to be conclusive, leaving enough place to conjecture about sizeable unequal $s$ and $\bar{s}$ distributions (see discussion in Refs. [6, 7]). Most recently, the E866 Collaboration [8] has measured a large $\bar{u}-\bar{d}$ asymmetry in the nucleon which may be succesfully accomodated in the framework of a pion cloud model.

The question of a non-perturbative asymmetry in the nucleon sea has been addressed by a number of authors. The most significant framework to discuss this issue appears to be the Meson Cloud Model (MCM). In this context, it is assumed that the nucleon can fluctuate to a meson-baryon bound state with a small probability. Non-perturbative sea-quark distributions are then associated with the valence quark and anti-quark distributions inside the baryon and the meson respectively. A first attempt in this direction was carried out in [6] where the nucleon-meson-baryon vertex is parameterized with ad-hoc form factors. In Ref. [7], intrinsic sea quark and anti-quarks are rearranged with valence quarks using simple two-body wave functions for describing meson-baryon bound states. Finally, let us also mention a different approach to the problem, based on a generalized chiral Gross-Neveu model at large $N_{c}$ [9].

In this letter, we employ both effective and perturbative degrees of freedom and show that a well-known scheme related to hadron production in hadronic collisions, viz. recombination, may be succesfully applied for describing meson-baryon fluctuations yielding non-perturbative sea quark-antiquark asymmetries. Our description of the nucleon-meson-baryon vertex starts from the perturbative production of a $q \bar{q}$ pair out of a dressed valence quark. Such perturbative sea quarks subsequently rearrange with the remaining valence quarks via recombination, in order to give rise to a hadronic nucleon fluctuation. As we will see, this provides a phenomenologically motivated one-parameter dependent description of the low $Q^{2}$ scale meson and baryon probability distributions inside the nucleon. The non-perturbative contributions to the strange sea of the nucleon are then calculated by means of widely used convolution forms. We obtain a structured asymmetry indicating a clear excess of strange quarks (antiquarks) for large (small) momentum fraction, becoming negligible beyond $x \sim 0.8$.

\section{Strange meson-baryon fluctuation and $s-\bar{s}$ asym- metry in the nucleon}

We start by considering a simple picture of the nucleon in the infinite momentum frame as being formed by three dressed valence quarks - valons - which carry all of its momentum. The valon distribution in the nucleon has been calculated by Hwa 
in Ref. [10], and it has been found to be

$$
v(x)=\frac{105}{16} \sqrt{x}(1-x)^{2} .
$$

For the sake of simplicity, we will not distinguish between $u$ and $d$ valon densities, so identical momentum distribution will be assumed for both flavors.

In the framework of the MCM, the nucleon can fluctuate to a meson-hyperon bound state carrying zero net strangeness. As a first step in such a process, we may consider that each valon can emit a gluon which, before interacting, decays perturbatively into a $s \bar{s}$ pair. The probability of having such a perturbative $q \bar{q}$ pair can then be computed in terms of Altarelli-Parisi splitting functions [1]

$$
P_{g q}(z)=\frac{4}{3} \frac{1+(1-z)^{2}}{z}, \quad P_{q g}(z)=\frac{1}{2}\left(z^{2}+(1-z)^{2}\right) .
$$

These functions have a physical interpretation as the probability of gluon emision and $q \bar{q}$ creation with momentum fraction $z$ from a parent quark or gluon respectively. Hence,

$$
q\left(x, Q^{2}\right)=\bar{q}\left(x, Q^{2}\right)=N \frac{\alpha_{s t}^{2}\left(Q^{2}\right)}{(2 \pi)^{2}} \int_{x}^{1} \frac{d y}{y} P_{q g}\left(\frac{x}{y}\right) \int_{y}^{1} \frac{d z}{z} P_{g q}\left(\frac{y}{z}\right) v(z)
$$

is the joint probability density of obtaining a quark or anti-quark coming from subsequent decays $v \rightarrow v+g$ and $g \rightarrow q+\bar{q}$ at some fixed low $Q^{2}$. As the valon distribution does not depend on $Q^{2}$ [10], the scale dependence in eq. (3) only exhibits through the strong coupling constant $\alpha_{s t}$. The range of values of $Q^{2}$ at which the process of virtual pair creation occurs in our approach is typically about $1 \mathrm{GeV}^{2}$, as dictated by the valon model of the nucleon. For definiteness, we will use $Q=0.8 \mathrm{GeV}$ as in Ref. [10, for which $\alpha_{s t}^{2} \sim 0.3$ is still sufficiently small to allow for a perturbative evaluation of the $q \bar{q}$ pair production. Since the scale must be consistent with the valon picture, the value of $Q^{2}$ is not really free and cannot be used to control the flavor produced at the $g q \bar{q}$ vertex. Instead, this role can be ascribed to the normalization constant $N$, which must be such that to a heavier quark corresponds a lower value of $N$. For instance, it could be fixed by comparing the output of eq. (3) with fit analyses of experimental data of sea quark distributions in the nucleon at low $Q^{2}$. However, we will for simplicity include the whole factor appearing in eq. (3) in the global normalization of the $|M B\rangle$ Fock state.

Once a $s \bar{s}$ pair is produced, it can rearrange itself with the remaining valons so as to form a most energetically favored meson-baryon bound state. To obtain the meson and baryon probability densities inside the nucleon, one has to employ effective techniques in order to deal with the non-perturbative $Q C D$ processes inherent to the dressing of quarks into hadrons. Although in-nucleon meson and baryon are virtual states, one may assume that the mechanisms involved in their formation are similar to those at work in the production of real hadrons in hadronic collisions. 
Therefore we shall proceed to describe the $N \rightarrow M B$ fluctuation by means of a well-known recombination model approach [12].

Notice that as the nucleon fluctuates into a meson-baryon bound state, the meson and baryon distributions inside the nucleon are not independent. Actually, to ensure the zero net strangeness of the nucleon and momentum conservation, the in-nucleon meson and baryon distributions must fulfill two basic constraints:

$$
\int_{0}^{1} d x\left[P_{B}(x)-P_{M}(x)\right]=0, \quad \int_{0}^{1} d x\left[x P_{B}(x)+x P_{M}(x)\right]=1,
$$

which can be both guaranteed by choosing

$$
P_{M}(x)=P_{B}(1-x)
$$

for all momentum fractions $x$. In addition to satisfying conservation laws, condition (5) enables us to calculate just one of the two distributions. Therefore, we can proceed to compute the strange meson distribution $P_{M}(x)$ along the lines of Ref. [12] and then relate it to the hyperon probability as indicated in eq. (5). Using the recombination model, the probability density of having a meson out of two quarks is given by

$$
P_{M}(x)=\int_{0}^{x} \frac{d y}{y} \int_{0}^{x-y} \frac{d z}{z} F(y, z) R(x, y, z)
$$

where $F(y, z)$ is

$$
F(y, z)=\beta y v(y) z \bar{q}(z)(1-y-z)
$$

and $R(x, y, z)$ is the recombination function associated with the meson formation

$$
R(y, z)=\alpha \frac{y z}{x^{2}} \delta\left(1-\frac{y+z}{x}\right)
$$

The normalization of the meson distribution is not given by the recombination model. We shall thus fix the overall normalization as given by the experimental probability that the strange hadronic fluctuation occurs; this value is phenomenologically estimated to be about $4-10 \%$ (see e.g. Refs.[7, 13]). In Fig.1 we show our calculated meson and baryon probability densities inside the nucleon.

The non-perturbative strange and anti-strange sea distributions can be now computed by means of the two-level convolution formulas

$$
s^{N P}(x)=\int_{x}^{1} \frac{d y}{y} P_{B}(y) s_{B}(x / y) \quad \bar{s}^{N P}(x)=\int_{x}^{1} \frac{d y}{y} P_{M}(y) \bar{s}_{M}(x / y),
$$

where the sources $s_{B}(x)$ and $\bar{s}_{M}(x)$ are primarily the probability densities of the strange valence quark and anti-quark in baryon and meson respectively, evaluated at

\footnotetext{
${ }^{1}$ Although condition (5) has been generally employed (see e.g. Refs. [1, 13]), it is not a unique choice since eqs. (1) relate the integrals of the meson and baryon probability densities and not the distributions themselves.
} 
the hadronic scale $Q^{2}$ [6]. In principle, to obtain the non-perturbative distributions given by eqs. (9), one should sum over all the meson-hyperon fluctuations of the nucleon but, since such hadronic Fock states are necessarilly off-shell, the most likely configurations are those closest to the nucleon energy-shell, namely $\Lambda^{0} K^{+}, \Sigma^{+} K^{0}$ and $\Sigma^{0} K^{+}$, for a proton state. However, as we are using the same distribution for $u$ and $d$ valons, the three configurations above contribute in the same way to the strange non-perturbative structure of the nucleon. Other fluctuations involving heavier in-nucleon mesons and baryons should be strongly suppressed due to their high virtuality 1 .

As long as experimental measurements are lacking at present, several choices are possible for the $\bar{s}_{M}$ and $s_{B}$ distributions in $K$-ons and $\Lambda$ or $\Sigma$ baryons respectively. In this respect, it has been a common practice to employ modified light valence quark distributions of pions and protons [6, 13]. However, we think that one should better use simple forms reflecting the fact that strange valence quarks should carry a rather large amount of momentum in $S= \pm 1$ hadron states at low $Q^{2}$ scales. Indeed, the momentum distribution found by Shigetani et al. [14 in the framework of a Nambu-Jona Lasinio model at low $Q^{2}$, adequately reflects this feature (see also Ref. 15]). A similar form for the strange distribution in $K$-ons has been found very recently in [16], using a Monte Carlo based program for generating valence distributions in hadrons. We thus put forward the following simple form

$$
x \bar{s}_{M}(x)=6 x^{2}(1-x)
$$

which is an excellent approximation to these phenomenological data. For similar reasons, in $\Lambda$ and $\Sigma$ baryons we expect a $s_{B}$ momentum distribution peaked around $1 / 2$, which after normalization can be approximated by

$$
x s_{B}(x)=12 x^{2}(1-x)^{2} .
$$

For comparison, we have also considered other forms for $\bar{s}_{M}$ and $s_{B}$ in order to display their effect on the shape of the non-perturbative distributions of eqs. (9). As suggested in [6, 13], we have used $\bar{s}_{M}=(1-x)^{0.18} q^{\pi}$, where $q^{\pi}$ is the valence distribution in pions, and $s_{B}=u_{N} / 2$, with $u_{N}$ the up valence distribution in the nucleon; $q^{\pi}$ and $u_{N}$ are those given in Ref. 16. In Fig. 2 we show the $s^{N P}$ and $\bar{s}^{N P}$ distributions as well as the $s-\bar{s}$ asymmetry predicted within our model.

\section{Summary and discussion}

By means of an approach involving both effective and perturbative degrees of freedom, we have obtained $s$ and $\bar{s}$ non-perturbative distributions in the nucleon's sea.

We based our approach on a valon description of the initial state of the nucleon, which perturbatively produces sea quark/anti-quark pairs. Thereafter these quarks

\footnotetext{
${ }^{2}$ For instance, a loosely bound $p \phi$ configuration can be neglected since it is rather heavier than a $\Lambda K$ state and Zweig's rule suppressed.
} 
and anti-quarks give rise to a hadronic bound state by means of recombination with the remaining valons. An interesting feature of the model is that it allows a full representation of the non-perturbative processes inside the nucleon in terms of a well-known effective scheme of hadron physics [12. In this way, a specific connection between the physics of hadronic reactions and that of hadron fluctuations is established in the approach. This is a pleasant aspect of the approach since the same physical principles, related to hadronization, should be involved in both situations. The present model also exhibits a remarkable economy of parameters when compared to other approaches in the literature. Notice for instance that neither nucleon-meson-hyperon coupling constants nor vertex functions are used to recognize the extended nature of the nucleon-meson-hyperon vertex, avoiding the need of such parameters, whose values are still controversial.

As can be seen in Fig. 2, the model predicts a definite structured asymmetry in the strange sea of the nucleon. As we have discussed in the introduction, our results are compatible with the present status of experimental data coming from $\nu N$ and $\bar{\nu} N$ scattering, due to the large error bars allowed for such distributions [4]. One should however notice that the predicted non-perturbative $s^{N P}$ and $\bar{s}^{N P}$ distributions depend on the form of the strange and anti-strange distributions in the in-nucleon baryon and meson respectively. So, although the model is reliable in predicting different distributions for sea quarks and anti-quarks, their exact shapes cannot be determined until we have more confident results for quark densities inside strange hadrons. Nevertheless, the model clearly predicts an excess of sea quarks over anti-quarks carrying a large fraction of the nucleon's momentum (see Fig. 2) and this appears to be independent of the exact form of the strange and anti-strange distributions inside the in-nucleon baryon and meson. This result coincides with the predictions of Ref. [6, 0, 9] and strongly differs with the outcome of Ref. [13]. Our $s^{N P}$ and $\bar{s}^{N P}$ distributions, as well as the difference between them, are similar in shape and magnitude to those found in a recent analysis with a different approach based on two-body wave functions with a number of parameters [7]. Another point deserving a comment concerns the probability of the strange meson-baryon fluctuation. As far as we know, direct experimental information is still not available and one then has to rely on phenomenological estimates that it should range between 4 and $10 \%$.

It has been recently shown that double polarization observables in $\phi$ meson photoproduction off protons [17] are very sensitive to the strange quark content of the proton itself, indicating an interesting source of experimental information for giving further support to the current discussion.

The present analysis can be easily extended to study non-perturbative contributions to other flavors. A pion cloud version of the model should also predict a noticeable $\bar{u}-\bar{d}$ asymmetry in the sea of the nucleon, as announced by the recent data of the E866 Collaboration [8]. In this sense, it is worth to mention that the data analysis resulting from the above mentioned experiment favors an explanation in terms of the MCM rather than some alternative approaches. 


\section{Acknowledgments}

We acknowledge useful discussions with M. Malheiro. We also thank Centro Brasileiro de Pesquisas Físicas (CBPF) for the warm hospitality extended to us during this work. H.R.C. was partially supported by Centro Latino Americano de Fisica (CLAF). J.M. and H.R.C. are supported by Fundação de Amparo à Pesquisa do Estado de Rio de Janeiro (FAPERJ).

\section{References}

[1] W. Melnitchouk and A.W. Thomas, Phys. Lett. B414, 134 (1997).

[2] C. Adloff et al. (H1 Colaboration), Z. Phys. C74, 191 (1997).

[3] J. Breitweg et al. (ZEUS Collaboration), Z. Phys. C74, 207 (1997).

[4] A.O. Bazarko et al. (CCFR Collaboration), Z. Phys. C65, 189 (1995).

[5] J. Botts et al. (CTEQ Collaboration), Phys. Lett. 304, 159 (1993); H. Lai et al. Phys. Rev. D51, 4763 (1995).

[6] A. Signal and A.W. Thomas, Phys. Lett. B191, 205 (1987).

[7] S.J. Brodsky and B.Q. Ma, Phys. Lett. B381, 317 (1996). B.Q. Ma and S.J. Brodsky, SLAC-PUB-7501, hep-ph/9707408.

[8] E.A. Hawker et al. (E866/NuSea Collaboration), Phys. Rev. Lett 80, 3715 (1998); J.C. Peng et al. (E866/NuSea Collaboration), Phys. Rev. D58, 092004 (1998).

[9] M. Burkardt and and B.J. Warr, Phys. Rev. D45, 958 (1992).

[10] R.C. Hwa, Phys. Rev. D22, 759 (1980); ibid. 1593.

[11] G. Altarelli and G. Parisi, Nuc. Phys. B126, 298 (1977).

[12] K.P. Das and R.C. Hwa, Phys. Lett B68, 459 (1977).

[13] W. Melnitchouk and M. Malheiro, Phys. Rev. C55, 431 (1997).

[14] T. Shigetani, K. Suzuki and H. Toki, Phys. Lett. B308, 383 (1993).

[15] J.T. Londergan, G.Q. Liu and A.W. Thomas, Phys. Lett. B380, 393 (1996).

[16] A. Edin and G. Ingelman, Phys. Lett. B432, 402 (1998).

[17] A.I. Titov, Y. Oh, S.N. Yang and T. Morii, Phys. Rev. C58, 2429 (1998). 


\section{Figure Captions}

Fig. 1: Meson and baryon probability densities in the nucleon, normalized to 4\%. Full line is for the strange meson distribution in the nucleon. Dashed line shows the strange baryon distribution $P_{B}(x)=P_{M}(1-x)$.

Fig. 2: Upper: The non-perturbative strange distributions in the nucleon sea. Full lines are for $s^{N P}$ (thin) and $\bar{s}^{N P}$ (thick), as obtained by using eqs. (10) and (11). Point lines are for the non-perturbative distributions coming from the choice $s_{B}=u_{N} / 2$ (thin) and $\bar{s}_{M}=(1-x)^{0.18} q^{\pi}$ (thick).

Lower: The $s(x)-\bar{s}(x)$ asymmetry in the nucleon sea. The full line is the asymmetry obtained by using $s_{B}$ and $\bar{s}_{M}$ as given by eqs. (10) and (11), whereas the point line is the asymmetry coming from a $s_{B}=u_{N} / 2$ distribution in the hyperon and a $\bar{s}_{M}=(1-x)^{0.18} q^{\pi}$ in the kaon. 


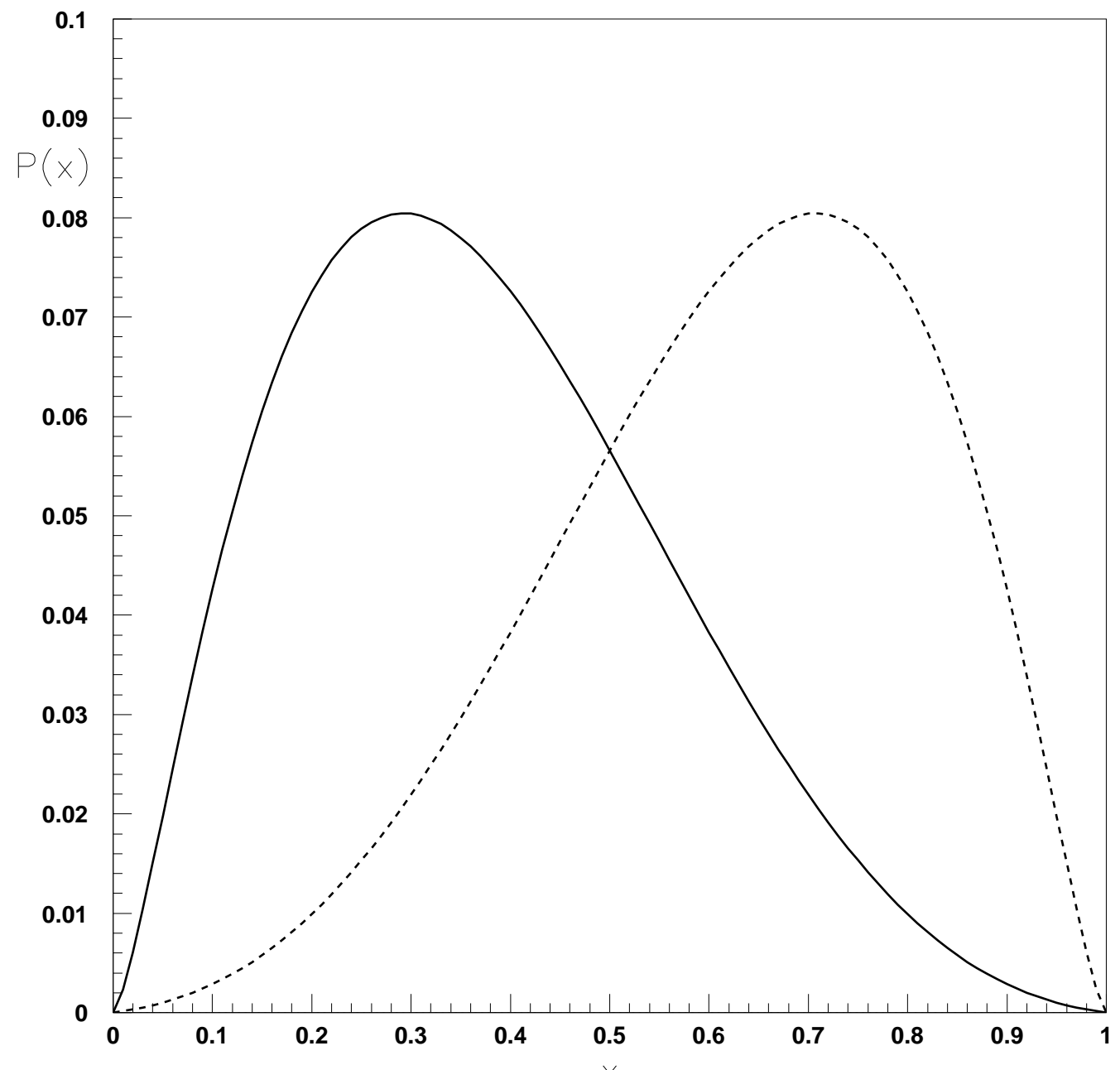

Figure 1: 


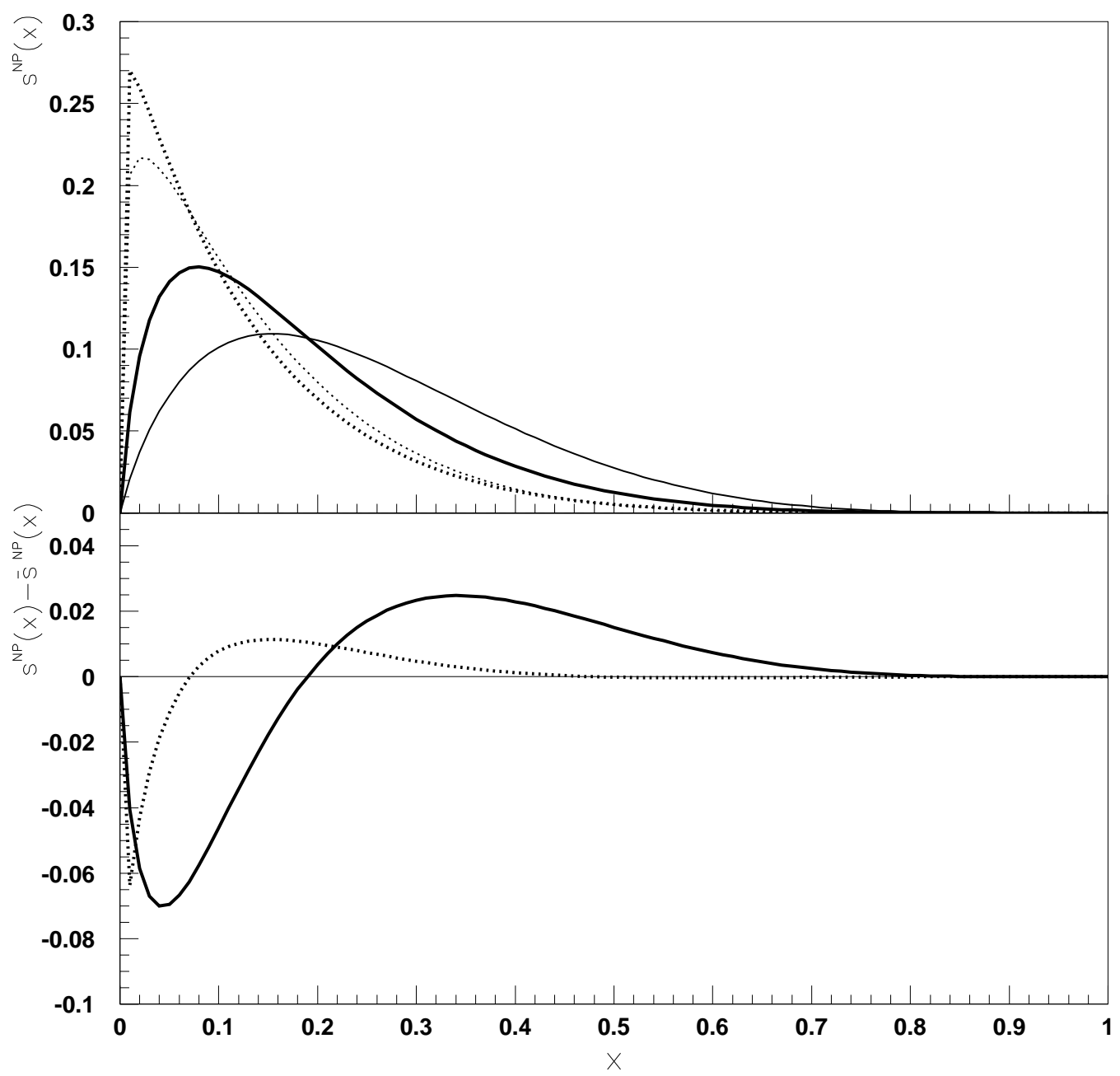

Figure 2: 